\title{
Neuropsychological Performance and Conversion to Alzheimer's Disease in Early-Compared to Late-Onset Amnestic Mild Cognitive Impairment: CREDOS Study
}

\author{
Byoung Seok Ye ${ }^{\mathrm{a}}$ Sang Won Seo ${ }^{\mathrm{a}}$ Yunhwan Lee ${ }^{\mathrm{c}}$ Seong Yoon Kim ${ }^{\mathrm{b}}$ \\ Seong Hye Choid Young Min Lee ${ }^{\mathrm{e}}$ Do Hoon Kim ${ }^{\mathrm{g}}$ Hyun Jeong Han ${ }^{\mathrm{h}}$ \\ Duk L. Na ${ }^{a}$ Eun-Joo Kim ${ }^{f}$
}

\begin{abstract}
aDepartment of Neurology, Samsung Medical Center, Sungkyunkwan University School of Medicine, and ${ }^{b}$ Department of Psychiatry, University of Ulsan College of Medicine, Asan Medical Center, Seoul, 'Departments of Preventive Medicine and Public Health, Ajou University School of Medicine, Suwon, dDepartment of Neurology, Inha University School of Medicine, Incheon, e Department of Psychiatry, Busan National University School of Medicine, and 'Department of Neurology, Pusan National University Hospital, Pusan National University School of Medicine and Medical Research Institute, Busan, ${ }^{9}$ Department of Psychiatry, Hallym University College of Medicine, Chuncheon, and hyongji Hospital, Goyang, Republic of Korea
\end{abstract}

\section{Key Words}

Mild cognitive impairment $\cdot$ Alzheimer disease $\cdot$ Age of onset $\cdot$ Neuropsychological tests $\cdot$ Disease progression

\begin{abstract}
Background: Amnestic mild cognitive impairment (aMCI) is regarded as a prodromal stage of Alzheimer's disease (AD). Given that patients with early-onset $A D$ (EOAD) and with late-onset $A D$ (LOAD) are known to have different clinical courses, symptoms and neuroimaging findings, early-onset $(\mathrm{EOMCl})$ and late-onset aMCI (LOMCI) might be expected to have similar differences as EOAD versus LOAD. Methods: Our study involving 425 patients with aMCl (124 EOMCl, 301 LOM$\mathrm{Cl}$ ), who were followed for around 1.5 years, and 958 normal control subjects (NC) investigated neuropsychological char-
\end{abstract}

acteristics and prediction of progression to $A D$ in patients with $\mathrm{EOMCl}$ versus $\mathrm{LOMCI}$. Neuropsychological scores were compared between $\mathrm{EOMCl}, \mathrm{LOMCl}$ and $\mathrm{NC}$ with analyses of covariance controlling age, gender, education and disease duration. The risk of AD conversion was evaluated by Cox proportional hazard analyses. Results: The baseline neuropsychological performances were comparable between $\mathrm{EOMCl}$ and $\mathrm{LOMCl}$. Visuospatial memory for $\mathrm{EOMCl}$ and verbal memory scores for LOMCI were significant predictors of AD conversion. Conclusion: Our study indicates that $\mathrm{EOMCI}$ with visuospatial memory impairment, which implies underlying right predominant pathology, and LOMCI with poor verbal memory, which suggests underlying left predominant pathology, are individual conditions at an increased risk of conversion to AD.

Copyright $\odot 2012$ S. Karger AG, Basel

\section{KARGER}

Fax +4161306 1234

E-Mail karger@karger.ch

www.karger.com
(C) 2012 S. Karger AG, Basel

$1420-8008 / 12 / 0344-0156 \$ 38.00 / 0$

Accessible online at:

www.karger.com/dem
Eun-Joo Kim

Department of Neurology, Pusan National University Hospita

Pusan National University School of Medicine and Medical Research Institute

1-10 Ami-dong, Seo-gu, Busan, 602-739 (Republic of Korea)

E-Mail eunjookim@pusan.ac.kr 


\section{Introduction}

Alzheimer's disease (AD) is the most common cause of neurodegenerative dementia, which usually begins with prominent memory impairment and progresses to visuospatial, language and frontal executive dysfunctions [1]. These clinical features are in accordance with the hierarchical progression of AD pathology [2].

Most patients with AD develop their disease after the age of 65 , but $1-6 \%$ of all $\mathrm{AD}$ patients have a disease onset before the age of 65 [3]. Some researchers arbitrarily classify $\mathrm{AD}$, with a cut-off age of 65 years, into early-onset (EOAD) and late-onset $\mathrm{AD}$ (LOAD). Although both subtypes are known to have the same histopathological features [4], different phenotypic presentations and rates of cognitive decline have frequently been noted. Cortical dysfunctions including language problem, visuospatial dysfunction or apraxia are more prominent in EOAD [5, 6], while predominant memory dysfunction is more common in LOAD [7]. In addition, EOAD has a more rapid progression of cognitive and functional declines than LOAD $[6,8]$.

Recent neuroimaging studies revealed that functional or structural changes in EOAD were greater in magnitude and extent than those of LOAD, particularly in the temporoparietal junction, posterior cingulate cortex and precuneus $[8,9]$, while LOAD had more prominent medial temporal involvements than those in $\operatorname{EOAD}[10,11]$. These neuroimaging observations are compatible with different clinical characteristics between EOAD and LOAD, as described above.

However, regarding structural or functional hemispheric asymmetry between EOAD and LOAD, there have been inconsistent results. Some studies found that EOAD demonstrated more prominent language dysfunctions $[6,12,13]$ and more severe hypometabolism in the left hemisphere $[14,15]$. However, others revealed that EOAD showed greater deficits in visuospatial and attentional performances [16] and more severe hypometabolism in the right temporoparietal regions [17-19]. Likewise, no consistent functional and cognitive hemispheric asymmetry has been seen in LOAD [20,21].

Amnestic mild cognitive impairment (aMCI) is referred to as a prodromal stage of AD [22]. Patients with aMCI show memory dysfunction with preserved functional abilities in activities of daily living (ADL) and 10$20 \%$ of aMCI annually progresses into AD [23, 24]. Considering that aMCI is a transitional state between normal aging and $\mathrm{AD}$, it might be speculated that aMCI can also be divided into early-onset (EOMCI) and late-onset
aMCI (LOMCI), according to the age of symptom onset. Given that EOAD and LOAD have different clinical features and anatomical substrates, EOMCI and LOMCI might be expected to have similar patterns of differences to those between EOAD and LOAD.

To our knowledge, there has only been one study investigating differences in neuropsychological characteristics and cerebral metabolic patterns between EOMCI and LOMCI [25]. The 5-year longitudinal follow-up study showed that EOMCI patients had better performances only in verbal recall and word fluency tests, even though they had more extensive hypometabolism than LOMCI patients [25]. However, it might be difficult to generalize from their results because of the small number of patients. Moreover, the authors could not compare the rate of conversion to $\mathrm{AD}$ between EOMCI and LOMCI due to the small sample size.

Thus, in this study involving a large sample of patients with aMCI who were followed for around 1.5 years, we investigated: (1) differences in baseline neuropsychological characteristics between EOMCI and LOMCI, (2) differences in the rate of conversions to $\mathrm{AD}$ between $\mathrm{EOMCI}$ and LOMCI, (3) differences in baseline neuropsychological performances between converters (CVs) and nonconverters (NCVs) in each aMCI group, (4) differences in baseline neuropsychological performances between CVs of EOMCI (EOCVs) and LOMCI (LOCVs), and finally, (5) baseline neuropsychological test predicting $\mathrm{AD}$ progression in EOMCI and LOMCI subjects, respectively. We hypothesized that the EOMCI group might have more rapid progression than the LOMCI group and that the baseline neuropsychological performances of EOCVs and LOCVs, even at the stage of aMCI, might reflect the neuropsychological characteristics of EOAD and LOAD. Based on our hypothesis, we presumed that a baseline neuropsychological test predicting $\mathrm{AD}$ progression would be different between EOMCI and LOMCI.

\section{Materials and Methods}

This study was conducted as part of the Clinical Research Center for Dementia of South Korea (CREDOS) study, which is a multicenter hospital-based prospective cohort study. From May 2005 to April 2010, a total of 1,749 subjects with aMCI and 958 subjects with normal cognition (NC) were consecutively recruited in 31 memory disorder clinics at university-based hospitals. All aMCI patients met the following criteria proposed by Petersen [26]: (1) subjective memory complaint by patients or caregivers, (2) normal general cognitive function as defined by scores on the Korean version of the Mini-Mental State Examination (MMSE) $[27] \geq-1.0 \mathrm{SD}$ of the norms for age- and education-matched nor- 
mal subjects, (3) normal ADL, as judged both clinically and on the ADL scale described below, (4) objective memory decline below the 16 th percentile $(-1.0 \mathrm{SD})$ on either verbal or visual memory tests, and (5) not demented.

All of the patients underwent comprehensive interviews, neurological examinations and neuropsychological assessment as described in the previous study [28]. In brief, caregivers were interviewed in depth by neurologists or psychiatrists and neuropsychologists. Patients with current or past neurological or psychiatric illnesses such as schizophrenia, epilepsy, brain tumors, encephalitis and severe head trauma were excluded. Subjects with physical illnesses that could interfere with the clinical study, such as hearing or vision loss, aphasia, severe cardiac disorders, severe respiratory illnesses, malignancy and hepatic or renal disorders were excluded. Blood tests for all participants included a complete blood count, blood chemistry tests, vitamin $\mathrm{B}_{12} /$ folate, syphilis serology and thyroid functioning tests. Apolipoprotein $\mathrm{E}$ (ApoE) genotype was determined by polymerase chain reaction, as explained in the previous CREDOS study [29]. Conventional brain MRI scans confirmed the absence of structural lesions such as tumors, traumatic brain injuries, hydrocephalus or severe white matter hyperintensities. Among the 1,749 aMCI subjects, there were 142 subjects with severe white matter hyperintensities. These subjects were excluded due to the inconclusive possibility that they might have vascular rather than degenerative MCI. Twenty subjects with other causes of cognitive decline ( 9 subjects with a clinical diagnosis of vascular MCI having relevant focal neurologic signs, 8 subjects with cerebral infarction, 3 subjects with cerebral hemorrhage and 1 subject with vitamin $B_{12}$ deficiency) were also excluded from further research. As a result, there were 1,587 aMCI subjects with putative degeneration, who were divided into two subgroups, according to the age when memory symptoms began. Patients with onset at age $<65$ years were classified as EOMCI, whereas those with onset age $\geq 65$ years were classified as LOMCI. Among 1,587 aMCI subjects, 81 subjects with uncertain age of onset were additionally excluded. The remaining 1,506 aMCI subjects were grouped, on the basis of an onset age of 65 years, as 465 EOMCI (30.9\%) and 1,041 LOMCI (69.1\%).

The 958 subjects of the NC group who visited a memory clinic to check their cognitive functions had neither a history of neurological or psychiatric disease. Of the NC group, 891 subjects presented with a subjective memory complaint and the remaining 67 with a subjective non-memory complaint.

\section{Clinical Evaluations}

We used the Dementia Evaluation Package developed by CREDOS, which is composed of the Clinical Evaluation Form and the Caregiver Questionnaire Form, as described in a previous study [28]. The Clinical Evaluation Form includes each of the following: (1) the history of cognitive decline from the caregiver, (2) the MMSE [30], (3) the Clinical Dementia Rating (CDR) and CDR Sum of Boxes score (CDR-SOB) [31], (4) the Global Deterioration Scale [32], (5) the Hachinski ischemia scale [33], (6) a neurological examination, and (7) the Geriatric Depression Scale (GDS) [34]. The Caregiver Questionnaire Form includes each of the following: (1) basic demographic data about the patient and caregiver, (2) lifestyle and family history, (3) past medical history, including vascular risk factors, (4) the Korean Dementia Screening Questionnaire [35], (5) the Barthel ADL index [36], (6) Caregiver ad- ministered Neuropsychiatric Inventory [37], and (7) the Seoul Instrumental ADL (S-IADL) [38]. To precisely evaluate the age of symptom onset, we asked subjects when their memory symptom began and confirmed it with the subjects' caregivers.

At the beginning of the CREDOS study, a symposium was convened in order to standardize diagnostic assessment and to ascertain inter- and intra-center reliability for clinical evaluation of patients participating in this study. Subsequently, the neurologists, psychiatrists, research nurses and psychologists met once every month in order to verify the quality of clinical data throughout the course of the study. The institutional review boards at all participating centers approved this study. Written, informed consent was obtained from patients and caregivers.

\section{Neuropsychological Assessment}

All subjects underwent a standardized neuropsychological battery known as the Seoul Neuropsychological Screening Battery, described in detail elsewhere [39]. The scoreable tests were comprised of the Digit Span (forward and backward), the Korean version of the Boston Naming Test [40], the Rey-Osterrieth Complex Figure Test (RCFT; which involves copying, immediate and 20-min delayed recall, and recognition), the Seoul Verbal Learning Test (SVLT; which involves three learning-free recall trials of 12 words, a 20 -min delayed recall trial of these 12 items, and a recognition test), phonemic and semantic Controlled Oral Word Association Tests (COWAT), and the Stroop Test (which involves word and color reading of 112 items during a period of $2 \mathrm{~min}$ ). Age-, sex- and education-specific norms for each test, based on 447 normal subjects, were used for comparison. Scores lower than -1.0 SD of the age-, sex- and education-adjusted norms were considered abnormal.

\section{Follow-Up}

Of the 1,506 aMCI subjects who met the criteria for aMCI, 427 patients who completed at least one follow-up visit with the same interview and neuropsychological tests for the baseline evaluation from May 2005 to February 2011 were initially included in the study. Two LOMCI whose follow-up diagnoses were dementia with Lewy bodies and frontotemporal dementia were excluded. Thus, a total of 425 aMCI subjects were included for the final analysis, of whom 124 (29.2\%) were EOMCI and 301 (70.8\%) were LOMCI, respectively. In this nationwide study, patients who completed follow-up neuropsychological tests were only enrolled for the longitudinal study, which explains the relatively low retention rate of the study. Basic demographic data of EOMCI, LOMCI and $\mathrm{NC}$ are presented in online supplementary table 1 (for all online suppl. material, see www.karger.com/doi/10.1159/000342973).

The mean duration of follow-up was $17.9 \pm 9.8$ months (range 4.2-53.8). Subjects who performed at least one follow-up neuropsychological test had a longer duration of education $(\mathrm{p}<0.001)$, higher baseline MMSE score $(\mathrm{p}=0.040)$ and lower depression scale score $(p=0.021)$ than those who did not. Although statistical significance was not reached, subjects with follow-up tended to be older $(\mathrm{p}=0.069)$, to have a lower proportion of female subjects $(\mathrm{p}=0.056)$ and to have lower baseline CDR-SOB scores ( $\mathrm{p}=$ $0.059)$ than those without. The proportion of follow-up subjects was not different between EOMCI and LOMCI (124/465, 26.7\%, vs. 301/1,039, 29.0\%; $\mathrm{p}=0.359$ ) (online suppl. table 2).

Logistic regression analysis using age, gender, education, baseline MMSE score, GDS and baseline CDR-SOB score as indepen- 
dent variables revealed that older age $[95 \% \mathrm{CI}$ of relative risk (RR) $1.00-1.03 ; \mathrm{p}=0.032]$ and longer duration of education $(95 \% \mathrm{CI}$ $1.03-1.09 ; \mathrm{p}<0.001$ ) were only associated with follow-up visit, being independent of other variables.

The diagnosis of dementia was based on criteria from the Diagnostic and Statistical Manual of Mental Disorders (4th edition) and required clinical evidence of cognitive deficits confirmed by neuropsychological tests, as well as evidence of impairment in social or occupational functions confirmed by ADL scales. For the diagnosis of probable $\mathrm{AD}$, we used the criteria of the National Institute of Neurological and Communicative Disorders and Stroke-Alzheimer's Disease and Related Disorders Association [4]. Among 425 finally included aMCI subjects, 124 progressed onto $\mathrm{AD}$ and 32 reverted to a state of normal cognition (better than -1.0 SD for age, gender and education matched norms in all cognitive domains).

\section{Statistical Analyses}

We used analyses of variance or $\chi^{2}$ tests to determine group differences in demographic and clinical characteristics. Age, gender, education and duration of symptoms were included as covariates for analyses of covariance (ANCOVA), in comparison of baseline neuropsychological test performances among EOMCI, LOM$\mathrm{CI}$ and NC. Cox proportional hazard models were used to illustrate the difference in converting to AD between EOMCI and LOMCI, after controlling for gender, education, baseline MMSE scores and duration of symptoms. Age was not included as a covariate which highly correlated with age at symptom onset $(\mathrm{R}=0.97, \mathrm{p}<0.001$ for Pearson's correlation analysis) and subtypes of aMCI (EOMCI vs. LOMCI; $\mathrm{R}=0.76, \mathrm{p}<0.001$ for Spearman's correlation analysis). Time to the event was defined as the time from study entry to the follow-up visit at which a first-time diagnosis of $\mathrm{AD}$ was made. Subjects that did not convert to AD were treated as censored observations from the time of their final follow-up evaluation.

To find the best neuropsychological test for predicting $\mathrm{AD}$ progression in EOMCI and LOMCI, we performed separate Cox proportional hazard analyses for each EOMCI and LOMCI subjects. In this analysis, we used age, gender, education, duration of symptoms and each neuropsychological test score at baseline evaluation.

\section{Results}

\section{Demographic and Clinical Characteristics}

The NC group was younger than the LOMCI group, but older than the EOMCI group. The EOMCI group had a significantly younger age of symptom onset than the LOMCI group. The EOMCI group had significantly longer disease duration than the LOMCI group. There were no significant differences in sex, education, GDS and the mean duration of follow-up between LOMCI and EOMCI groups. ApoE genotyping was performed in 74 of 124 EOMCI subjects (59.7\%), 181 of 301 LOMCI subjects (60.1\%) and 540 of $958 \mathrm{NC}$ subjects (56.4\%). EOMCI (47.3\%) and LOMCI (41.5\%) had a higher proportion of $\varepsilon 4$ allele carriers than NC (21.8\%). The proportion of $\varepsilon 4$ carriers was not different between EOMCI and LOMCI, while subjects with the $\varepsilon 4$ homozygote were more common in the EOMCI group (14.9\%) than in the LOMCI group (2.8\%) (online suppl. table 1$)$.

\section{Differences in Baseline Neuropsychological}

Performances between EOMCI, LOMCI and NC

ANCOVA for neuropsychological test results adjusted for age, gender, education and duration of symptoms showed that EOMCI and LOMCI subjects had poorer performance than $\mathrm{NC}$ in all neuropsychological tests (table 1). The LOMCI group had significantly lower scores in RCFT recognition and the Stroop color reading tests than the EOMCI group.

\section{Conversion to AD in EOMCI and LOMCI}

The ratio of $\mathrm{AD}$ CVs was higher in the LOMCI group than in the EOMCI group (33.6 vs. $18.5 \%$; $=0.002$ ). Annual conversion rates were $11.5 \% / y e a r$ in EOMCI and 23.3\%/year in LOMCI, respectively. Cox analysis adjusting gender, education, duration of symptoms and baseline MMSE score revealed that the risk of AD conversion was higher in LOMCI than in EOMCI [hazard ration $(\mathrm{HR})=1.79,95 \%$ CI 1.10-2.91; $\mathrm{p}=0.02$ ] (fig. 1).

\section{Demographic Characteristics and Baseline \\ Neuropsychological Test Results between CVs and \\ NCVs in Each aMCI Group}

The independent $t$ test was used to compare the demographic characteristics and neuropsychological test performances between CVs and NCVs in total aMCI, EOMCI and LOMCI groups (table 2). The aMCI CVs were older and had a longer duration of education than aMCI NCVs. The proportion of $\varepsilon 4$ allele carriers was significantly higher in CVs than in NCVs $(48 / 85,56.5 \%$, vs. $62 / 170,36.5 \% ; p=0.003)$. The sex ratio was not different between CVs and NCVs. CVs had significantly lower scores on all measures of verbal and visual memory and executive function abilities than NCVs. CVs had significantly lower MMSE scores and higher baseline CDR-SOB scores than those of NCVs.

EOCVs did not differ in age and sex ratio from NCVs of the EOMCI group (EONCVs). However, EOCVs had a longer duration of education and a higher proportion of $\varepsilon 4$ allele carriers $(14 / 20,70.0 \%$, vs. $21 / 54,38.9 \%$; $p=0.021)$ than those of EONCVs. EOCVs had poorer performances in RCFT immediate recall and RCFT delayed recall tests than EONCVs. MMSE and CDR-SOB scores were not different between EOCVs and EONCVs. 
Table 1. Comparison of baseline neuropsychological test results between EOMCI, LOMCI and NC after adjustment for age, gender, education and duration of symptoms

\begin{tabular}{|c|c|c|c|c|}
\hline $\begin{array}{l}\text { Neuropsychological test } \\
\text { (possible maximum score) }\end{array}$ & $\begin{array}{l}\text { EOMCI } \\
(\mathrm{n}=124)\end{array}$ & $\begin{array}{l}\text { LOMCI } \\
(\mathrm{n}=301)\end{array}$ & $\begin{array}{l}\text { NC } \\
(n=958)\end{array}$ & $\mathrm{p}$ value \\
\hline \multicolumn{5}{|l|}{ Attention } \\
\hline Digit span backward & $3.3(0.1)^{\mathrm{a}}$ & $3.5(0.1)^{\mathrm{a}}$ & $3.9(0.04)$ & $<0.001$ \\
\hline \multicolumn{5}{|l|}{ Language } \\
\hline K-BNT (60) & $40.7(0.8)^{\mathrm{a}}$ & $39.7(0.5)^{\mathrm{a}}$ & $47.5(0.3)$ & $<0.001$ \\
\hline \multicolumn{5}{|l|}{ Memory } \\
\hline SVLT immediate recall (36) & $14.4(0.4)^{\mathrm{a}}$ & $14.4(0.3)^{\mathrm{a}}$ & $19.5(0.2)$ & $<0.001$ \\
\hline SVLT delayed recall (12) & $2.5(0.2)^{\mathrm{a}}$ & $2.4(0.1)^{\mathrm{a}}$ & $6.3(0.1)$ & $<0.001$ \\
\hline SVLT recognition score $(24)$ & $18.1(0.2)^{\mathrm{a}}$ & $18.0(0.1)^{\mathrm{a}}$ & $20.7(0.1)$ & $<0.001$ \\
\hline RCFT immediate recall (36) & $7.4(0.6)^{\mathrm{a}}$ & $8.0(0.4)^{\mathrm{a}}$ & $16.0(0.2)$ & $<0.001$ \\
\hline \multicolumn{5}{|l|}{ Frontal/executive function } \\
\hline COWAT supermarket & $12.3(0.6)^{\mathrm{a}}$ & $12.2(0.4)^{\mathrm{a}}$ & $16.8(0.2)$ & $<0.001$ \\
\hline COWAT phonemic & $17.1(1.1)^{\mathrm{a}}$ & $18.0(0.7)^{\mathrm{a}}$ & $25.3(0.4)$ & $<0.001$ \\
\hline Stroop color reading test (112) & $71.9(2.2)^{\mathrm{a}, \mathrm{b}}$ & $65.7(1.4)^{\mathrm{a}, \mathrm{b}}$ & $84.6(0.8)$ & $<0.001$ \\
\hline MMSE score (30) & $25.3(0.3)^{\mathrm{a}}$ & $25.0(0.2)^{\mathrm{a}}$ & $27.5(0.1)$ & $<0.001$ \\
\hline
\end{tabular}

LOCVs did not differ in age, sex ratio and education from NCVs of the LOMCI group (LONCVs). The proportion of $\varepsilon 4$ allele carriers was higher in LOCVs than in LONCVs (34/65, 52.3\%, vs. 41/116, 35.3\%; p = 0.029). LOCVs scored lower than LONCVs in SVLT delayed recall, COWAT supermarket item and MMSE score. The CDR-SOB score was significantly higher in LOCVs than in LONCVs. Although statistical significance was not reached, LOCVs tended to have lower baseline scores in the Korean version of the Boston Naming Test (K-BNT), the SVLT recognition score and the COWAT animal test than LONCVs.

\section{Comparison of Baseline Neuropsychological Test}

Results between EOCV, LOCV and NC Groups

Baseline neuropsychological performances were compared among EOCV, LOCV and NC groups by ANCOVA using age, gender, education and duration of symptoms as covariates (table 3). Both the EOCV and LOCV groups showed worse performances than the NC group in almost all neuropsychological tests. EOCVs scored lower in the RCFT immediate recall test when compared to LOCVs. There was no tests in which LOCVs had a significantly lower score compared to EOCVs.

\section{Neuropsychological Predictors of AD Conversion in EOMCI and LOMCI}

Table 4 shows Cox proportional hazard regression analyses with adjustment for potential confounders including age, gender, education and duration of symptoms for the association between baseline neuropsychological test results and $\mathrm{AD}$ conversion in EOMCI and LOMCI. In the EOMCI group, lower scores in K-BNT, RCFT immediate recall, RCFT delayed recall and MMSE were associated with a higher risk of $\mathrm{AD}$ conversion. On the other hand, the LOMCI group had a higher risk of $\mathrm{AD}$ conversion when the scores of SVLT immediate recall, SVLT delayed recall, RCFT recognition score, COWAT supermarket, Stroop color reading test and MMSE were lower or the scores in CDR-SOB were higher in 
Table 2. Comparison of demographic characteristics and baseline neuropsychological results between CVs and NCVs among total aMCI, EOMCI and LOMCI

\begin{tabular}{|c|c|c|c|c|c|c|c|c|c|}
\hline & \multicolumn{3}{|l|}{ Total aMCI } & \multicolumn{3}{|l|}{ EOMCI } & \multicolumn{3}{|l|}{ LOMCI } \\
\hline & CVs & NCVs & $\mathrm{p}$ value & EOCVs & EONCVs & $\mathrm{p}$ value & LOCVs & LONCVs & $\mathrm{p}$ value \\
\hline Subjects & 124 & 301 & & 23 & 101 & & 101 & 200 & \\
\hline \multicolumn{10}{|l|}{ Demographic features } \\
\hline $\begin{array}{l}\text { Age at baseline evaluation, } \\
\text { years }\end{array}$ & $72.2(7.5)$ & $69.8(7.6)$ & 0.002 & $60.9(6.5)$ & $61.7(5.2)$ & 0.507 & $74.8(4.8)$ & $73.8(4.9)$ & 0.090 \\
\hline Women & $49[39.5]$ & $119[39.5]$ & $>0.999$ & $12[52.2]$ & $62[61.4]$ & 0.483 & $63[62.4]$ & 118 [59.9] & 0.708 \\
\hline Education, years & $9.9(4.9)$ & $8.7(5.0)$ & 0.023 & $11.0(3.7)$ & $9.0(4.4)$ & 0.047 & $9.7(5.1)$ & $8.6(5.2)$ & 0.084 \\
\hline ApoE $\varepsilon 4$ allele dose & 85 & 170 & & 20 & 54 & & 65 & 116 & \\
\hline 0 & $37[43.5]$ & $108[63.5]$ & 0.002 & $6[30.0]$ & $33[61.1]$ & 0.017 & $31[47.7]$ & 75 [64.7] & ] 0.026 \\
\hline 1 & $36[42.4]$ & $58[34.1]$ & 0.199 & $7[35.0]$ & $17[31.5]$ & 0.774 & $29[44.6]$ & $41[35.3]$ & ] 0.219 \\
\hline 2 & $12[14.1]$ & $4[2.4]$ & 0.001 & $7[35.0]$ & $4[7.4]$ & 0.007 & $5[7.7]$ & 0 & 0.005 \\
\hline \multirow{2}{*}{\multicolumn{10}{|c|}{$\begin{array}{l}\text { Neuropsychological test (possible maximum score) } \\
\text { Attention }\end{array}$}} \\
\hline & \multicolumn{7}{|c|}{ Attention } & & \\
\hline Digit span forward & $5.5(1.5)$ & $5.4(1.7)$ & 0.737 & $5.9(1.9)$ & $5.7(1.9)$ & 0.698 & $5.3(1.4)$ & $5.2(1.5)$ & 0.471 \\
\hline Digit span backward & $3.3(0.9)$ & $3.3(1.1)$ & 0.709 & $3.5(0.9)$ & $3.5(1.3)$ & 0.981 & $3.2(0.9)$ & $3.2(1.0)$ & 0.988 \\
\hline \multicolumn{10}{|l|}{ Language } \\
\hline K-BNT (60) & $37.0(9.5)$ & $39.4(9.3)$ & 0.027 & $42.0(10.0)$ & $42.0(8.9)$ & 0.987 & $35.7(9.0)$ & $38.0(9.2)$ & 0.066 \\
\hline \multicolumn{10}{|l|}{ Visuospatial function } \\
\hline RCFT copy (36) & $29.2(6.9)$ & $28.6(7.6)$ & 0.470 & $29.9(6.4)$ & $30.1(6.0)$ & 0.880 & $29.0(7.0)$ & $27.9(8.1)$ & 0.222 \\
\hline \multicolumn{10}{|l|}{ Memory } \\
\hline SVLT immediate recall (36) & $13.8(4.0)$ & $14.3(4.0)$ & 0.180 & $15.7(4.4)$ & $15.1(3.7)$ & 0.474 & $13.3(3.8)$ & $14.0(4.1)$ & 0.195 \\
\hline SVLT delayed recall (12) & $1.6(1.9)$ & $2.6(2.2)$ & $<0.001$ & $2.2(2.2)$ & $3.0(2.3)$ & 0.119 & $1.4(1.7)$ & $2.3(2.1)$ & $<0.001$ \\
\hline SVLT recognition score $(24)$ & $17.4(2.6)$ & $18.0(2.4)$ & 0.031 & $18.1(3.1)$ & $18.3(2.6)$ & 0.763 & $17.3(2.5)$ & $17.8(2.4)$ & 0.050 \\
\hline RCFT immediate recall (36) & $5.6(4.2)$ & $7.1(5.5)$ & 0.002 & $5.0(4.7)$ & $8.6(6.4)$ & 0.004 & $5.7(4.1)$ & $6.4(4.8)$ & 0.248 \\
\hline RCFT delayed recall (36) & $5.2(4.3)$ & $7.0(5.2)$ & 0.001 & $5.4(5.3)$ & $8.8(5.6)$ & 0.009 & $5.2(4.1)$ & $6.0(4.7)$ & 0.148 \\
\hline RCFT recognition score (24) & $17.2(2.6)$ & $17.8(2.4)$ & 0.023 & $18.1(1.9)$ & $18.4(2.4)$ & 0.537 & $17.0(2.7)$ & $17.5(2.4)$ & 0.105 \\
\hline \multicolumn{10}{|l|}{ Frontal/executive function } \\
\hline COWAT animal & $11.1(4.3)$ & $12.0(3.8)$ & 0.045 & $13.1(3.9)$ & $12.8(3.3)$ & 0.755 & $10.7(4.3)$ & $11.5(3.9)$ & 0.086 \\
\hline COWAT supermarket & $11.1(4.2)$ & $12.8(5.2)$ & 0.001 & $13.5(4.4)$ & $13.5(5.2)$ & 0.974 & $10.5(4.0)$ & $12.4(5.2)$ & 0.001 \\
\hline COWAT phonemic & $17.7(9.1)$ & $17.2(9.5)$ & 0.696 & $23.1(9.7)$ & $18.4(9.8)$ & 0.046 & $16.4(8.4)$ & $16.6(9.3)$ & 0.818 \\
\hline Stroop color reading test (112) & $61.8(23.8)$ & $67.4(25.3)$ & 0.038 & $74.1(21.4)$ & $78.1(23.1)$ & 0.452 & $58.8(23.5)$ & $61.8(24.6)$ & 0.318 \\
\hline Geriatric depression scale (30) & $13.0(7.3)$ & $13.8(8.0)$ & 0.337 & $12.8(8.0)$ & $15.3(8.0)$ & 0.180 & $13.1(7.1)$ & $13.1(8.0)$ & 0.997 \\
\hline MMSE score (30) & $24.2(3.3)$ & $25.3(3.3)$ & 0.003 & $24.7(2.8)$ & $25.9(2.8)$ & 0.091 & $24.1(3.5)$ & $25.0(3.5)$ & 0.038 \\
\hline CDR-SOB score & $1.8(0.9)$ & $1.5(0.8)$ & 0.001 & $1.7(0.8)$ & $1.4(0.8)$ & 0.158 & $1.8(0.9)$ & $1.6(0.9)$ & 0.007 \\
\hline
\end{tabular}

Data are expressed as the mean, with SD in parenthesis; figures in brackets are percentages.

baseline neuropsychological tests. To further analyze the most predictive neuropsychological test for AD conversion, we performed Cox proportional hazard analyses entering age, gender, education, duration of symptoms and prior neuropsychological measures significantly predicting $\mathrm{AD}$ conversion. In the EOMCI group, since RCFT immediate recall and RCFT delayed recall $(\mathrm{R}=$ $0.888, \mathrm{p}<0.001)$, and K-BNT and MMSE $(\mathrm{R}=0.530, \mathrm{p}<$ 0.001 ) were significantly correlated, only two variables from each pair were entered in the analysis. Among the four combinations of variables, entering RCFT delayed recall and K-BNT gave the best fit model. Thus, both RCFT delayed recall $(\mathrm{RR}=0.89,95 \%$ CI $0.80-0.99 ; \mathrm{p}=$ $0.033)$ and $\mathrm{K}-\mathrm{BNT}(\mathrm{RR}=0.92,95 \%$ CI $0.86-0.98 ; \mathrm{p}=$ 0.009 ) were the best predictors of $\mathrm{AD}$ conversion in the EOMCI group. In the LOMCI group, SVLT delayed recall, RCFT recognition score, COWAT supermarket, Stroop color reading, MMSE and CDR-SOB were entered in the Cox model with adjustment for age, gender, education and duration of symptoms. Only the CDR-SOB was 


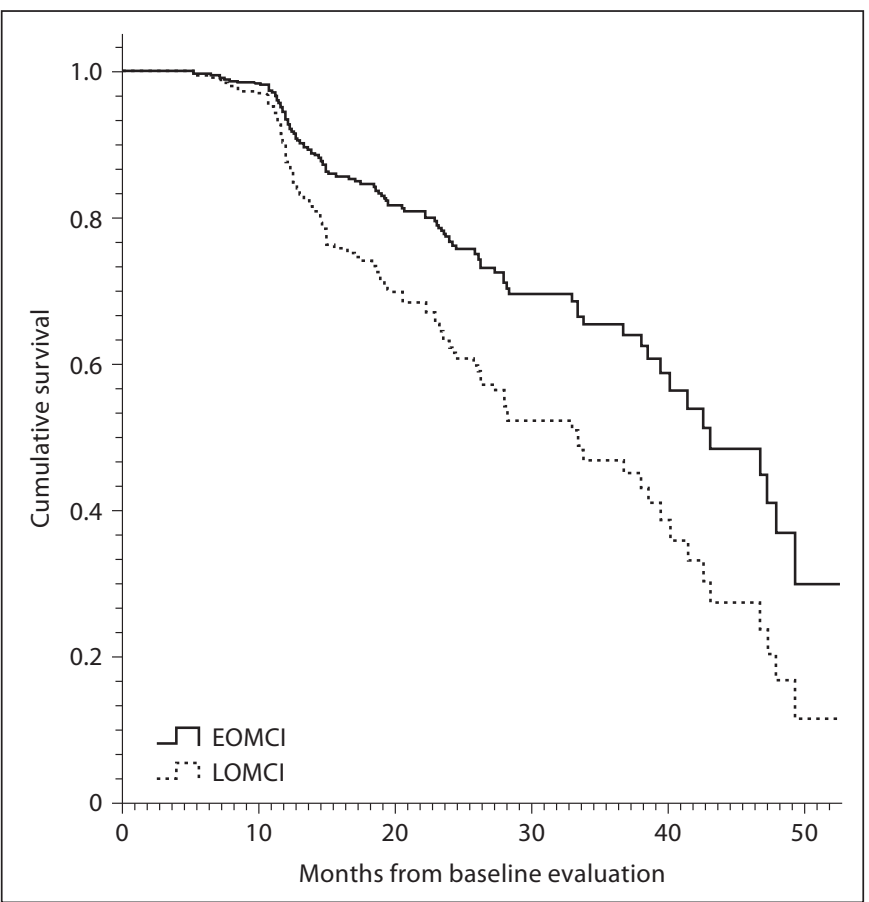

Fig. 1. Survival curves comparing cumulative proportions of subjects with EOMCI ( $<65$ years of age) and LOMCI ( $\geq 65$ years of age) who did not progress to $\mathrm{AD}$. Curves are based on the Cox proportional hazard analysis using gender, education, duration of symptoms and baseline MMSE score as covariates. The HR of AD conversion in subjects with LOMCI was 1.79 times higher than the HR in subjects with EOMCI (95\% CI 1.10-2.91; $\mathrm{p}=0.02)$.

a significant predictor of $\mathrm{AD}$ conversion $(\mathrm{RR}=1.32,95 \%$ CI 1.01-1.74; $\mathrm{p}=0.044)$. When the general cognitive indices such as MMSE and CDR-SOB scores were excluded from the model, SVLT delayed recall best predicted future $\mathrm{AD}$ conversion $(\mathrm{RR}=0.86,95 \%$ CI $0.76-0.99 ; \mathrm{p}=$ $0.031)$ in LOMCI.

\section{Discussion}

In this study, we tried to find the effects of age of onset on the pattern of demographic characteristics and neuropsychological profiles in aMCI. We found that there were no significant differences in demographic factors including sex ratio, education and ratio of the $\varepsilon 4$ allele carrier between EOMCI and LOMCI. The proportions of ApoE $\varepsilon 4$ carriers in aMCI and the control group were generally similar to those observed in other prospective studies [41, 42]. However, both the EOMCI and LOMCI groups showed a higher ratio of the $\varepsilon 4$ allele carrier than the $\mathrm{NC}$ group, and the ratio of subjects having the $\varepsilon 4$ homozygote was significantly higher in the EOMCI group than in the LOMCI group. In addition, CVs had a higher proportion of the $\varepsilon 4$ allele carriers than NCVs in both EOMCI and LOMCI groups. These results are consistent with previous reports suggesting that the $\varepsilon 4$ allele was a definite biological risk factor for $\mathrm{AD}$ and may increase the risk of developing EOAD $[43,44]$. One recent study revealed that the frequency of $\varepsilon 4$ homozygotes was highest in the group of $\mathrm{AD}$ with an age at onset of 60-69 years which may further support our result of a higher frequency of $\varepsilon 4$ homozygote in the EOMCI group [45].

The annual conversion rate of aMCI patients was 19.6\%/year. The Cox proportional hazard analysis adjusting gender and education revealed that the risk of $\mathrm{AD}$ conversion in EOMCI was lower than that in LOMCI. To our knowledge, this is the first study comparing the annual AD conversion rate between EOMCI and LOMCI. Even though one previous study with 5-year follow-up of EOMCI and LOMCI showed the annual conversion rate of the total MCI group (11.5\%), the authors did not investigate the annual conversion rate for each EOMCI and LOMCI group, due to the small number of subjects [25]. However, considering the relatively short duration of follow-up in our study, it could be cautiously interpreted that EOMCI progresses relatively slower than LOMCI.

An important finding of this study was that neuropsychological prediction of conversion to $\mathrm{AD}$ was different between EOMCI and LOMCI (table 4). Subjects with more impairment in naming, visuospatial memory and MMSE had an increased risk of AD conversion in the EOMCI group compared to subjects with more impairment in verbal memory, visuospatial recognition, semantic word generation, MMSE and CDR-SOB in the LOMCI group. In the EOMCI group, visuospatial memory and the confrontational naming test were the best neuropsychological predictors of time for conversion to AD. However, unlike in the EOMCI group, the general cognitive index, i.e. CDR-SOB score or SVLT delayed recall, was a highly significant predictor of future clinical deterioration in the LOMCI group. The predictive value of mainly verbal memory impairments for the LOMCI group in this study may be consistent with previous studies examining neuropsychological markers for predicting conversion to dementia in aMCI, in which verbal or episodic memory deficit was consistently found to have predictive power in aMCI subjects whose mean age was in their 70s [46, 47]. These results may suggest that those who are at a high risk of AD conversion from both EOMCI and LOMCI might 
Table 3. Comparison of baseline neuropsychological test results between EOCVs, LOCVs and NC

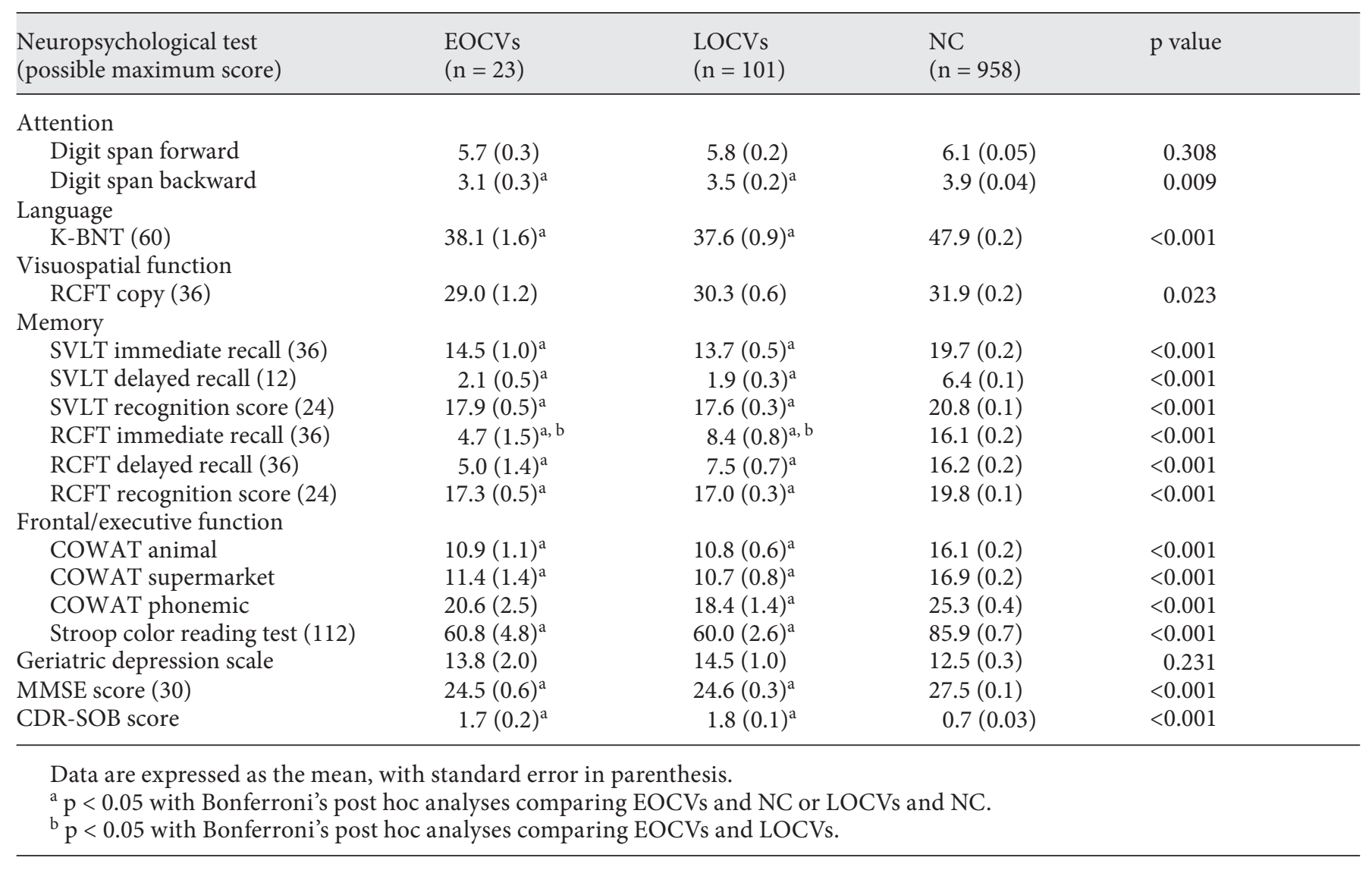

have, even in the aMCI stage, the distinguishing features of neuropsychological differences between EOAD and LOAD.

Lastly, regarding the comparison of baseline neuropsychological performances between CVs and NCVs, as we expected, the CVs showed worse performances in all tests than NCVs. However, the neuropsychological profiles with poor performance were different between EOCVs and LOCVs (table 2). That is, EOCVs were more impaired in visual memory tests than EONCVs, while LOCVs were more impaired in the verbal memory and semantic word generation test than LONCVs. Also, the EOCV group scored lower in immediate recall of visual memory than LOCVs. Even though there have been controversial results about hemispheric asymmetry between EOAD and LOAD, a few clinical and neuroimaging studies reported that EOAD had greater impairment in the right hemisphere than in the left [19] and LOAD had leftsided predominance $[20,21]$. Therefore, these different neuropsychological profiles of EOCVs (possibly right predominance) and LOCVs (possibly left predominance) in our study might reflect the fact that there are different asymmetric involvements of $\mathrm{AD}$ pathology between EOAD and LOAD $[8,18,19]$. One previous study found that LOCVs exhibited a worse performance on the visual memory test than LONCVs, while there were no tests showing a difference between EOCVs and EONCVs [25]. However, given the small sample size of the study, the results would be insufficient to detect any statistical certainty.

There are several limitations to this study. Firstly, histopathologic confirmation was not performed. Although we excluded subjects with clinical features of vascular MCI, frontotemporal dementia or dementia with Lewy bodies, there remains a possibility that the CVs of our study might have non-AD pathology [48]. Future study using Pittsburgh Compound B position emission tomography might be helpful in overcoming this limitation. 
Table 4. Relative risk of AD conversion associated with each baseline neuropsychological test result in EOMCI and LOMCI

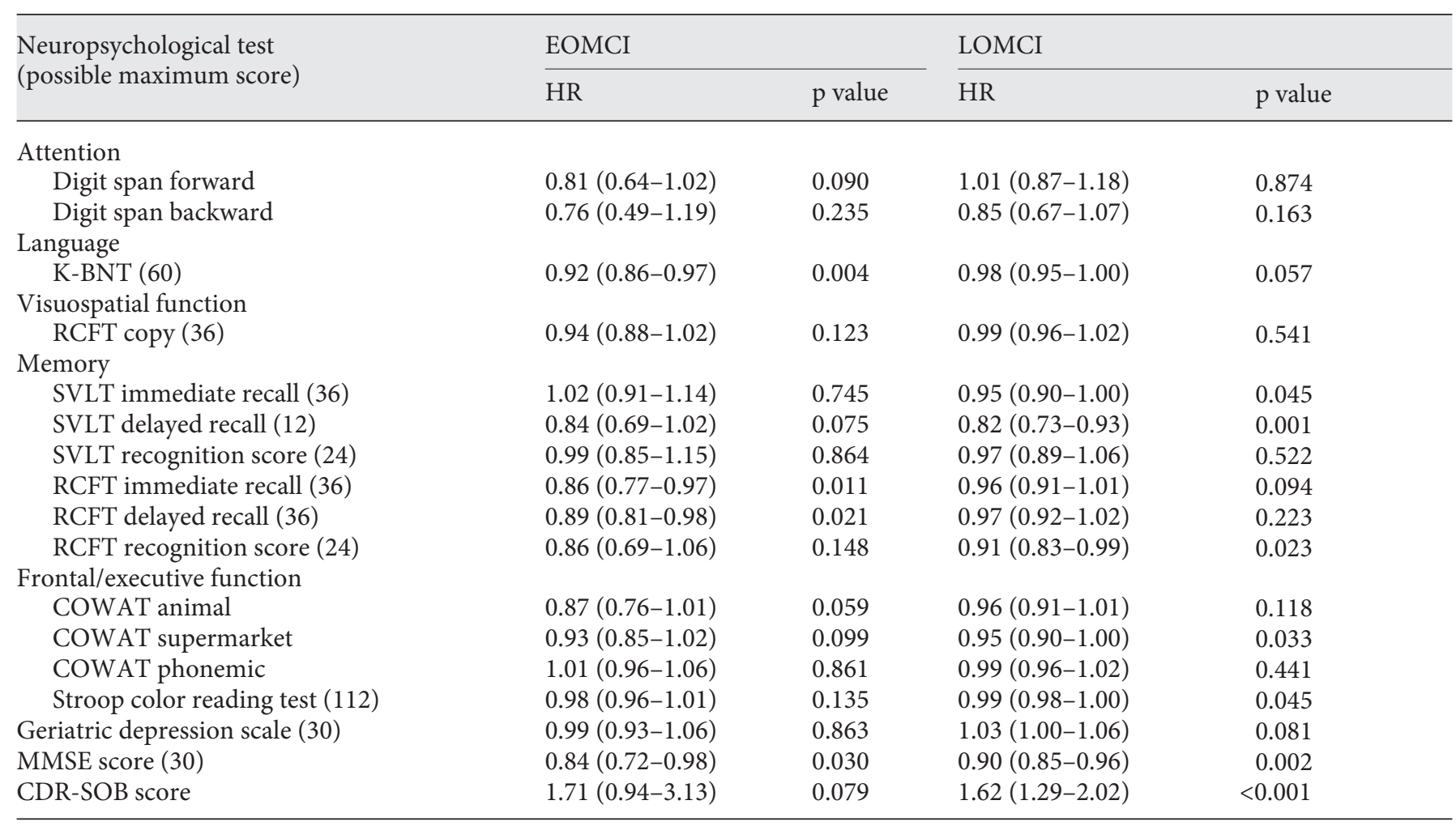

Figures in parentheses are $95 \%$ CIs.

Secondly, subjects with neuropsychological follow-up were significantly older and had longer durations of education than those without. Although we adjusted these variables to the analysis, there might be a selection bias. However, the proportion of neuropsychological followup or not was not different between EOMCI and LOMCI (online suppl. table 2). Thirdly, we could not test the effect or the interaction with another variable by ApoE genotype, due to the high proportion of missing values (about 40\%). ApoE genotype has a well-documented association with rates of progression in MCI subjects. Future study with thorough ApoE genotyping may be necessary to cover this topic.

\section{Acknowledgments}

This study was supported by a grant of the Korea Healthcare technology R\&D Project, Ministry of Health and Welfare, Republic of Korea (A102065).

References
1 Hodges JR: Alzheimer's centennial legacy: origins, landmarks and the current status of knowledge concerning cognitive aspects. Brain 2006;129:2811-2822.

$\checkmark 2$ Braak H, Braak E: Neuropathological stageing of Alzheimer-related changes. Acta Neuropathol 1991;82:239-259.

3 Bird TD: Alzheimer Disease Overview. Seattle, Departments of Neurology and Medicine, University of Washington, 1993.

-4 McKhann G, Drachman D, Folstein M, Katzman R, Price D, Stadlan EM: Clinical diagnosis of Alzheimer's disease: report of the NINCDS-ADRDA Work Group under the auspices of Department of Health and $\mathrm{Hu}$ man Services Task Force on Alzheimer's Disease. Neurology 1984;34:939-944.

$\checkmark 5$ Chui HC, Teng EL, Henderson VW, Moy AC: Clinical subtypes of dementia of the Alzheimer type. Neurology 1985;35:1544-1550.

6 Seltzer B, Sherwin I: A comparison of clinical features in early- and late-onset primary degenerative dementia. One entity or two? Arch Neurol 1983;40:143-146.
$\mathrm{Ye} / \mathrm{Seo} / \mathrm{Lee} / \mathrm{Kim} / \mathrm{Choi} / \mathrm{Lee} / \mathrm{Kim} / \mathrm{Han} / \mathrm{Na} /$ Kim 
7 Binetti G, Magni E, Padovani A, Cappa SF, Bianchetti A, Trabucchi M: Neuropsychological heterogeneity in mild Alzheimer's disease. Dementia 1993;4:321-326.

$\checkmark 8$ Kim EJ, Cho SS, Jeong Y, Park KC, Kang SJ, Kang E, Kim SE, Lee KH, Na DL: Glucose metabolism in early onset versus late onset Alzheimer's disease: an SPM analysis of 120 patients. Brain 2005;128:1790-1801.

9 Rabinovici GD, Furst AJ, Alkalay A, Racine CA, O’Neil JP, Janabi M, Baker SL, Agarwal N, Bonasera SJ, Mormino EC, Weiner MW, Gorno-Tempini ML, Rosen HJ, Miller BL, Jagust WJ: Increased metabolic vulnerability in early-onset Alzheimer's disease is not related to amyloid burden. Brain 2010;133:512-528.

$>10$ Kemp PM, Holmes C, Hoffmann SM, Bolt L, Holmes R, Rowden J, Fleming JS: Alzheimer's disease: differences in technetium $-{ }^{99} \mathrm{~m}$ HMPAO SPECT scan findings between early onset and late onset dementia. J Neurol Neurosurg Psychiatry 2003;74:715-719.

- 11 Frisoni GB, Pievani M, Testa C, Sabattoli F, Bresciani L, Bonetti M, Beltramello A, Hayashi KM, Toga AW, Thompson PM: The topography of grey matter involvement in early and late onset Alzheimer's disease. Brain 2007;130:720-730.

$\checkmark 12$ Filley CM, Kelly J, Heaton RK: Neuropsychologic features of early- and late-onset Alzheimer's disease. Arch Neurol 1986;43: 574-576.

-13 Koss E, Edland S, Fillenbaum G, Mohs R, Clark C, Galasko D, Morris JC: Clinical and neuropsychological differences between patients with earlier and later onset of Alzheimer's disease: a CERAD analysis, part XII. Neurology 1996;46:136-141.

-14 Yasuno F, Imamura T, Hirono N, Ishii K, Sasaki M, Ikejiri Y, Hashimoto M, Shimomura T, Yamashita H, Mori E: Age at onset and regional cerebral glucose metabolism in $\mathrm{Al}$ zheimer's disease. Dement Geriatr Cogn Disord 1998;9:63-67.

15 Small GW, Kuhl DE, Riege WH, Fujikawa DG, Ashford JW, Metter EJ, Mazziotta JC: Cerebral glucose metabolic patterns in Alzheimer's disease. Effect of gender and age at dementia onset. Arch Gen Psychiatry 1989; 46:527-532.

-16 Loring DW, Largen JW: Neuropsychological patterns of presenile and senile dementia of the Alzheimer type. Neuropsychologia 1985; 23:351-357.

17 Grady CL, Haxby JV, Horwitz B, Berg G, Rapoport SI: Neuropsychological and cerebral metabolic function in early vs late onset dementia of the Alzheimer type. Neuropsychologia 1987;25:807-816.

18 Ichimiya A, Herholz K, Mielke R, Kessler J, Slansky I, Heiss WD: Difference of regional cerebral metabolic pattern between presenile and senile dementia of the Alzheimer type: a factor analytic study. J Neurol Sci 1994;123:11-17.
19 Koss E, Friedland RP, Ober BA, Jagust WJ: Differences in lateral hemispheric asymmetries of glucose utilization between earlyand late-onset Alzheimer-type dementia. Am J Psychiatry 1985;142:638-640.

20 Baron JC, Chetelat G, Desgranges B, Perchey G, Landeau B, de la Sayette V, Eustache F: In vivo mapping of gray matter loss with voxelbased morphometry in mild Alzheimer's disease. Neuroimage 2001;14:298-309.

21 Giannakopoulos P, Hof PR, Bouras C: Alzheimer's disease with asymmetric atrophy of the cerebral hemispheres: morphometric analysis of four cases. Acta Neuropathol 1994;88:440-447.

22 Dubois B, Feldman HH, Jacova C, Cummings JL, Dekosky ST, Barberger-Gateau P, Delacourte A, Frisoni G, Fox NC, Galasko D, Gauthier S, Hampel H, Jicha GA, Meguro K, O'Brien J, Pasquier F, Robert P, Rossor M, Salloway S, Sarazin M, de Souza LC, Stern Y, Visser PJ, Scheltens P: Revising the definition of Alzheimer's disease: a new lexicon. Lancet Neurol 2010;9:1118-1127.

23 Farias ST, Mungas D, Reed BR, Harvey D, DeCarli C: Progression of mild cognitive impairment to dementia in clinic- vs community-based cohorts. Arch Neurol 2009;66: 1151-1157.

24 Petersen RC, Roberts RO, Knopman DS, Boeve BF, Geda YE, Ivnik RJ, Smith GE, Jack CR Jr: Mild cognitive impairment: ten years later. Arch Neurol 2009;66:1447-1455.

25 Kim SH, Seo SW, Yoon DS, Chin J, Lee BH, Cheong HK, Han SH, Na DL: Comparison of neuropsychological and FDG-PET findings between early- versus late-onset mild cognitive impairment: a five-year longitudinal study. Dement Geriatr Cogn Disord 2010;29: 213-223.

26 Petersen RC: Mild cognitive impairment as a diagnostic entity. J Intern Med 2004;256: 183-194.

27 Kang Y, Na DL, Hahn S: A validity study on the Korean Mini-Mental State Examination (K-MMSE) in dementia patients. J Korean Neurol Assoc 1997;15:300-307.

28 Park HK, Na DL, Han SH, Kim JY, Cheong $\mathrm{HK}$, Kim SY, Hong CH, Kim DK, Ku BD, Moon SY, Lee JY, Shim YS, Youn YC, Kim EJ, Kim BC, Park KH, Cha KR, Seo SW, Lee JH: Clinical Characteristics of a Nationwide Hospital-Based Registry of Mild-to-Moderate Alzheimer's Disease Patients in Korea: a CREDOS (Clinical Research Center for Dementia of South Korea) Study. J Korean Med Sci 2011;26:1219-1226.

29 Hong YJ, Yoon B, Shim YS, Cho AH, Shin HE, Kim YI, Kim SY, Yang DW: ApoE epsilon4 allele status in Korean dementia patients with severe white matter hyperintensities. J Alzheimers Dis 2011;24:519-524.
30 Han C, Jo SA, Jo I, Kim E, Park MH, Kang Y: An adaptation of the Korean Mini-Mental State Examination (K-MMSE) in elderly Koreans: demographic influence and population-based norms (the AGE study). Arch Gerontol Geriatr 2008;47:302-310.

31 Choi SH, Lee BH, Kim S, Hahm DS, Jeong JH, Yoon SJ, Jeong Y, Ha CK, Nab DL: Interchanging scores between clinical dementia rating scale and global deterioration scale. Alzheimer Dis Assoc Disord 2003;17:98-105.

32 Reisberg B, Ferris SH, de Leon MJ, Crook T: The Global Deterioration Scale for assessment of primary degenerative dementia. Am J Psychiatry 1982;139:1136-1139.

33 Pantoni L, Inzitari D: Hachinski’s ischemic score and the diagnosis of vascular dementia: a review. Ital J Neurol Sci 1993;14:539-546.

- 34 Yesavage JA, Brink TL, Rose TL, Lum O, Huang V, Adey M, Leirer VO: Development and validation of a geriatric depression screening scale: a preliminary report. J Psychiatr Res 1982;17:37-49.

-35 Ahn HJ, Chin J, Park A, Lee BH, Suh MK, Seo SW, Na DL: Seoul Neuropsychological Screening Battery-dementia version (SNSBD): a useful tool for assessing and monitoring cognitive impairments in dementia patients. J Korean Med Sci 2010;25:1071-1076.

36 Sinoff G, Ore L: The Barthel Activities of Daily Living Index: self-reporting versus actual performance in the old-old ( $>$ or $=75$ years). J Am Geriatr Soc 1997;45:832-836.

37 Kang SJ, Choi SH, Lee BH, Jeong Y, Hahm DS, Han IW, Cummings JL, Na DL: Caregiver-Administered Neuropsychiatric Inventory (CGA-NPI). J Geriatr Psychiatry Neurol 2004;17:32-35.

$38 \mathrm{Ku}$ HM, Kim J, Kwon EJ, Kim SH, Lee HS, Ko $\mathrm{H}$ : A study on the reliability and validity of Seoul-Instrumental Activities of Daily Living (S-IADL). J Korean Neuropsychiatr Assoc 2004;43:189-199.

39 Kang Y, Na DL: Seoul Neuropsychological Screening Battery (SNSB). Incheon, Human Brain Research \& Consulting, 2003.

$40 \mathrm{Kim}$ H, Na DL: Normative data on the Korean version of the Boston Naming Test. J Clin Exp Neuropsychol 1999;21:127-133.

- 41 Risacher SL, Shen L, West JD, Kim S, McDonald BC, Beckett LA, Harvey DJ, Jack CR Jr, Weiner MW, Saykin AJ: Longitudinal MRI atrophy biomarkers: relationship to conversion in the ADNI cohort. Neurobiol Aging 2010;31:1401-1418.

42 Ellis KA, Bush AI, Darby D, De Fazio D, Foster J, Hudson P, Lautenschlager NT, Lenzo N, Martins RN, Maruff P, Masters C, Milner A, Pike K, Rowe C, Savage G, Szoeke C, Taddei $\mathrm{K}$, Villemagne V, Woodward M, Ames D: The Australian Imaging, Biomarkers and Lifestyle (AIBL) study of aging: methodology and baseline characteristics of 1,112 individuals recruited for a longitudinal study of Alzheimer's disease. Int Psychogeriatr 2009; 21:672-687. 
43 Farrer LA, Cupples LA, Haines JL, Hyman B, Kukull WA, Mayeux R, Myers RH, PericakVance MA, Risch N, van Duijn CM: Effects of age, sex, and ethnicity on the association between apolipoprotein E genotype and $\mathrm{Al}$ zheimer disease. A meta-analysis. ApoE and Alzheimer Disease Metaanalysis Consortium. JAMA 1997;278:1349-1356.

-44 van Duijn CM, de Knijff P, Cruts M, Wehnert A, Havekes LM, Hofman A, Van Broeckhoven C: Apolipoprotein E4 allele in a population-based study of early-onset Alzheimer's disease. Nat Genet 1994;7:74-78.
45 Davidson Y, Gibbons L, Pritchard A, Hardicre J, Wren J, Stopford C, Julien C, Thompson J, Payton A, Pickering-Brown SM, Pendleton N, Horan MA, Burns A, Purandare N, Lendon CL, Neary D, Snowden JS, Mann DM: Apolipoprotein E epsilon4 allele frequency and age at onset of Alzheimer's disease. Dement Geriatr Cogn Disord 2007;23. 60-66.

46 Sarazin M, Berr C, De Rotrou J, Fabrigoule C, Pasquier F, Legrain S, Michel B, Puel M, Volteau M, Touchon J, Verny M, Dubois B: Amnestic syndrome of the medial temporal type identifies prodromal AD: a longitudinal study. Neurology 2007;69:1859-1867.
7 Perri R, Serra L, Carlesimo GA, Caltagirone C: Amnestic mild cognitive impairment: difference of memory profile in subjects who converted or did not convert to Alzheimer's disease. Neuropsychology 2007;21:549-558.

48 Jicha GA, Parisi JE, Dickson DW, Johnson K, Cha R, Ivnik RJ, Tangalos EG, Boeve BF, Knopman DS, Braak H, Petersen RC: Neuropathologic outcome of mild cognitive impairment following progression to clinical dementia. Arch Neurol 2006;63:674-681. 